\title{
LA FRONTERA COMO INTERSTICIO. REFLEXIONES EN TORNO A LA VIOLENCIA EPISTÉMICA DE LAS FRONTERIZACIONES
}

\author{
The border as an interstice. \\ Reflections on epistemic violence of fronterizations
}

Fernanda Stang*

\begin{abstract}
Resumen. Al hilo de inquietudes surgidas en el trabajo de campo de una investigación sobre procesos migratorios de colombianos y peruanos con subjetivaciones sexo-genéricas no heteronormativas, el artículo problematiza las fronteras aparentemente claras trazadas entre los atributos definidos para seleccionar a los sujetos del estudio: la relación tiempoespacio implicada en el desplazamiento migratorio, y la subjetivación sexo-genérica "homosexual". A partir de esas reflexiones, y de aportes de autores que han trabajado en torno a la noción, se propone un modo intersticial de pensar la frontera.
\end{abstract}

Palabras clave: frontera; migraciones; violencia epistémica.

Abstract. In line with concerns emerged in the fieldwork of an investigation on migratory processes of Colombians and Peruvians with nonheteronormative sex-generic subjectivations, the article problematizes the apparently clear boundaries drawn between the attributes defined to select the subjects of the study: the relationship time-space involved in migratory displacement, and the "homosexual" sex-generic subjectivation. From these reflections, and from contributions from authors who have worked around the notion, an interstitial way of thinking about the border is proposed.

Keywords: border; migrations; epistemic violence.

\footnotetext{
Centro de Investigación en Ciencias Sociales y Juventud (CISJU), Universidad Católica Cardenal
} Raúl Silva Henríquez. Santiago, Chile. E-mail: fstang@ucsh.cl. Orcid: 0000-0003-3781-3024. 


\section{Introducción ${ }^{1}$}

...recuerdo que una vez, por ejemplo, le dije a mi mamá que estaba así como desesperada, que ya no quería que nadie me preguntara más si me iba a quedar en Chile porque yo no sabía qué responder, entonces como que mi mamá me dijo "pero ¿cuál es el problema?, si a usted le gustan los hombres, las mujeres, y también los trans, y no se hace problema por eso, ipor qué se va a hacer problema por vivir en dos países? (...) ¿quién dijo que uno sólo tenía que vivir en un solo país?, ¿quién dijo que uno no podía vivir en dos países? Y si eso es lo que le gusta a usted, ¿por qué se va a tener que quedar en uno solo?, si aquí la queremos y allá también. (Laura, segundo encuentro, julio de 2015)

Este fragmento de una de las entrevistas con Laura (colombiana, 38 años, 6 viviendo en Santiago al comenzar nuestros encuentros, en febrero de 2015) logra poner en escena una de las tensiones centrales que recorrió la investigación de la que surgen estas reflexiones, que se ocupó de procesos migratorios de colombianos y peruanos con subjetivaciones sexo-genéricas no heteronormativas ${ }^{2}$ residiendo en Santiago de Chile, al hilo de la pregunta sobre si se producen transformaciones en esos procesos de subjetivación por la llegada a una nueva configuración cultural nacional. Esa tensión, que constituye el tema central de este artículo, está representada por la arbitrariedad que subyace al trazado de fronteras entre categorías, implicado en la definición de lo que se entiende por migración, o en el intento de estabilizar una "identidad" sexual y de género, y con la violencia epistémica que está en su base. Este texto, entonces, se propone reflexionar en torno a este nudo temático, tomando como base el trabajo de campo desplegado en este estudio, para arribar a la propuesta de un modo de pensar la noción de frontera -construido a partir

1 Algunas ideas iniciales de este artículo fueron planteadas en una ponencia titulada "'Usted lo que quiere es clasificarme': algunas implicancias epistemológicas y éticas de la mirada performativa del género en un caso de investigación", presentada en el II Coloquio Internacional Saberes contemporáneos desde la diversidad sexual: teoría, crítica, praxis, realizado en Rosario (Argentina), el 27 y 28 de junio de 2013.

2 La idea de subjetivaciones sexo-genéricas no heteronormativas articula a su vez dos nociones: la de dispositivo sexo-género, y la de procesos de subjetivación. Con dispositivo sexo-género me refiero al repertorio de posiciones de sujetos sexuados y generizados con el que se nos interpela en una formación social específica, posiciones que son habitadas, moldeadas, resistidas o incluso trastocadas en el proceso de subjetivación. Este último concepto (proceso de subjetivación) alude a los distintos modos de habitar esas posiciones de sujeto, siempre atravesados por disputas. Entonces, cuando hablo de migrantes con subjetivaciones sexo-genéricas no heteronormativas me refiero a migrantes que, en ese dispositivo sexo-género, han estado ocupando posiciones que exceden la matriz binaria macho-varón / hembra-mujer (Stang, 2018). A su vez, se entiende por heteronormatividad el "principio organizador del orden de relaciones sociales, política, institucional y culturalmente reproducido, que hace de la heterosexualidad reproductiva el parámetro desde el cual juzgar (aceptar, condenar) la inmensa variedad de prácticas, identidades y relaciones sexuales, afectivas y amorosas existentes" (Pecheny, 2008, p. 14). Se trata, en definitiva, de "un dispositivo social conformado por prácticas y discursos que establecen a la heterosexualidad como categoría universal, natural y estable" (Hiller, 2012, p. 238). 
de una reapropiación de ideas de otros autores- que permita hacerse cargo, aunque sea en parte, de esa violencia.

Entonces, la idea es compartir ciertas aprehensiones sobre la forma en que lo observado en el trabajo de campo ha tensionado de modo insistente las fronteras aparentemente claras trazadas entre los atributos definidos para seleccionar a los sujetos de esta investigación: por un lado, la relación tiempo-espacio implicada en el desplazamiento migratorio, y por el otro, una subjetivación sexo-genérica "homosexual". En ambos casos, estas experiencias migratorias cuestionan el carácter estático de las categorías y, por lo tanto, desafían a pensar sobre las decisiones metodológicas y analíticas más apropiadas para intentar abordar estas ubicuidades.

Respecto del primero de los ejes, las experiencias de estos sujetos enmarañan el acá y el allá en idas y vueltas que diluyen la separación estática origen-destino, idas y vueltas que a su vez enredan temporalidades que impiden fijar plazos o marcar hitos o fechas claras que permitan hablar de inicios y duraciones fácilmente reconocibles de la experiencia migratoria. Se trata de trayectos y trayectorias que redefinen la idea de simultaneidad, a partir de la coexistencia de varios tiempos y lugares en una experiencia presente. Con relación al segundo de los ejes de análisis, las reflexiones a las que condujo el trabajo de campo giraron en torno a la dificultad de "operacionalizar" la multiplicidad y discontinuidad de las subjetivaciones sexo-genéricas, fluidez que a su vez se escenificaba en estas temporalidades y espacialidades que también se multiplicaban. El objetivo último de este artículo no es ofrecer respuestas acabadas a estas inquietudes, sino dejarlas planteadas como invitaciones para indagaciones futuras, de carácter sistemático, y, a la vez, aprovechar esas interrogantes para problematizar la idea de frontera -en sentido amplio- que atraviesa a ambas tensiones.

El trabajo de campo del que surgen estas inquietudes se realizó entre abril de 2014 y abril de 2016, aunque se aplicó una última entrevista en marzo de 2017, atendiendo al curso particular que había tomado una de las biografías migrantes del grupo de entrevistados. El escenario del trabajo, como ya se señaló, fue Santiago de Chile, y la muestra estuvo compuesta por siete personas migrantes de entre 19 y 38 años, con tiempos de residencia variables en la ciudad. Si se considera el nivel de escolaridad formal como indicio (proxy) de pertenencia de clase $^{3}$, los entrevistados pertenecen a lo

3 Con Bourdieu, entendemos a la clase como "un conjunto de agentes que, por el hecho de ocupar posiciones similares en el espacio social (eso es, en la distribución de poderes), están sujetos a condiciones de existencia y factores condicionantes similares, y, como resultado, están dotados de disposiciones similares que los dirigen a desarrollar prácticas similares" (Bourdieu, 1994). Esas posiciones similares están vinculadas a la disponibilidad de diversas formas de capital, y su distribución interna y externa. 
que, en términos amplios, puede definirse como clase media, desde una clase media baja hasta otra más "acomodada". El diseño metodológico se centró en la narrativa autobiográfica, y más específicamente, en la técnica del relato de vida. Se mantuvieron tres largos encuentros de conversación con cada uno de los entrevistados, y en algunos casos cuatro, más otros encuentros informales; también se realizaron 16 entrevistas a informantes clave, miembros de organizaciones de y para migrantes, y de organizaciones de la diversidad sexual.

\section{Ubicuidades y fronterizaciones}

La migración implica un trastocamiento de las dimensiones de espacio y tiempo (Benza, 2000; Gutiérrez Vidrio, 2015). En cierta forma, parece una afirmación tautológica, sin embargo, quienes estudiamos las migraciones no necesariamente nos hemos hecho cargo de sus implicancias, sobre todo en lo relativo a la dimensión temporal. Respecto de la dimensión espacial, ha habido desarrollos teóricos y metodológicos que, desde hace ya un tiempo, han procurado dar cuenta de este trastocamiento. Ciertamente, el enfoque transnacional (Levitt, Jaworsky, 2007; Khagram, Levitt, 2008) ha significado un importante aporte para que los estudios migratorios problematizaran la necesidad de hacerse cargo de la multiespacialidad del sujeto contemporáneo y de la experiencia humana, y de su vínculo con este tipo de procesos sociales en particular. A partir de algunas compilaciones sobre aspectos metodológicos de la investigación de las migraciones se han realizado llamados a complejizar la imagen de salida-llegada, pensando en cambio en "salidas-cruces-llegadas múltiples" (Velasco, Gianturco, 2012). Desde la demografía, tempranamente Domenach y Picouet (1990), a partir de sus reflexiones sobre la movilidad espacial de la población en relación a sus posibilidades de medición, proponían nociones como las de "residencia base" y "reversibilidad eventual de las migraciones", Ilamando la atención sobre el hecho que "el criterio de residencia única con todas sus implicancias territoriales, estadísticas, legales, inmobiliarias, socio-económicas, etc., es el que prevalece en el estudio de la migración" (Domenach, Picouet, 1990, p. 52), y enfatizando la inadecuación de este criterio a la realidad de las migraciones contemporáneas.

Aunque desde entonces ha pasado mucho, el enfoque transnacional entre otras cosas, la sentencia de los autores sigue teniendo validez. En general, seguimos hablando, por ejemplo, de países de origen y destino -agregando, sí, la categoría de países de tránsito-, o de migraciones de retorno. En nuestros análisis sobre las migraciones seguimos asumiendo un allá y un acá más o menos estables, aún incluso en los estudios multisituados, y las fronteras de los Estadosnación han jugado un rol central en este punto de vista generalizado. Esto es, en buena medida, inevitable, porque esas fronteras geopolíticas tienen una presencia ineludible en las vidas de los migrantes, con implicancias poderosas 
en su configuración cotidiana del espacio y el tiempo. Las fronteras del Estadonación son, en ese sentido, nudos decisivos en sus biografías (Levitt, Jaworkky, 2007), y las tramas narrativas de los entrevistados para el estudio del que surge esta reflexión son un ejemplo nítido, en la medida en que muchos de ellos construyen esa frontera como hito de un giro biográfico respecto de su subjetivación sexo-genérica. Pero, por lo mismo, no debiésemos asumir el Estadonación como una categoría natural y lógica de la organización de los procesos migratorios, sino construirla a partir de esas experiencias vitales, especialmente en investigaciones a escala microsocial.

También, por lo mismo, no debiésemos asumir que esa frontera geopolítica instaura de suyo un acá y un allá estables, homogéneos, e inmutables. El allá está presente en el acá (y ahora) del migrante, está encarnado en el estar acá, en una simultaneidad compleja, que puede adquirir diferentes formas, a las que como investigadores debiésemos aprender a estar atentos. La experiencia de Laura, condensada en la cita con la que abría este artículo, muestra la fluidez de esta experimentación del allá y el acá, y a la vez la tensión que le genera ser interpelada desde una imagen de la migración que estabiliza este movimiento, imagen instituida en el sentido común, también en buena parte del académico -un señalamiento del que, obviamente, no me exculpo-. Es cierto que esta fluidez está asociada con su pertenencia de clase, que como señalamos al principio, puede considerarse a grandes rasgos como clase media, y con el hecho de que, entre los países implicados en su migración (Colombia y Chile), el tránsito esté, aún con sus restricciones, al alcance, en términos de distancia geográfica y de acceso a la "legalidad" del traspaso, al menos para personas con sus características. Para una migrante peruana que se desempeñe en el trabajo doméstico puertas adentro, por ejemplo, esa fluidez de la circulación estaría eventualmente mucho más restringida -generalmente tienen "permiso" de sus empleadores para viajar a Perú cada dos años-. Pero la experiencia de Laura, por esa posición de relativo privilegio, intensifica una vivencia de simultaneidad encarnada que comparte con esa hipotética trabajadora de casa particular peruana.

Este efecto de intensificación, vinculado además con las particularidades del trabajo de Laura, permite observar con mayor claridad este anudamiento de espacios y tiempos, este continuum de la experiencia migratoria en el que el investigador introduce un corte de cuyas implicancias en su estudio debiera estar consciente. Ella ha organizado su vida laboral a partir de su vínculo con una organización lésbica de Colombia. Cuando decidió venir a Chile para convivir con Jaime, un trans chileno, activista, que conoció en un seminario en otro país latinoamericano, sus compañeras de la organización le propusieron seguir vinculada con ellas, a partir de su trabajo en proyectos que empezaron a pensar en clave regional, animadas por su decisión de migrar. Por esa razón, viaja con 
frecuencia a Colombia, en estancias de varios meses, y además se desplaza por otros países sudamericanos. Esta multiplicidad de trayectos -que también son tempos- incide de varias formas en la organización de su cotidianeidad, desde sus posibilidades de arrendar un espacio independiente para vivir -comparten un departamento con la mamá de Jaime- hasta en la ropa que va a comprar:

...obviamente también me encuentro con cosas prácticas como que, por ejemplo, hace rato con Jaime queremos independizarnos de su mamá, pero al mismo tiempo es como que nada, nos dicen "hay un apartamento" o "hay una casa", y él: "pero usted se va ya, y va a estar tanto tiempo afuera, y entonces no, más bien cuando vuelva", y siempre termina en esto, y yo misma digo "si nos vamos, pero yo no voy a estar, nos vamos a trastear y justo le toca quedarse solo", entonces como que a veces... o también cosas que acá me dicen, algunas personas sobre todo cuando estaba en la universidad: "mira, vamos a hacer un proyecto, iquieres participar?", y yo "sí, ¿cuándo es?", "si nos sale sería de tal a tal fecha", "ah, es que ahí no estoy". Y en Colombia [sucede] lo mismo, en Colombia [te dicen]: " $i O$ sea que regresaste a Bogotá? iMirá!, hay un trabajo perfecto para ti", "no, es que todavía sigo en Chile", "ah" (...) igual yo sí quiero tener una casa, igual sí necesito a veces cosas tan simples como pensar, no sé, por ejemplo, te digo algo súper cotidiano, re [muy] simple, tengo que comprarme un abrigo nuevo para el invierno, pero ahorita ya me voy a ir para Bogotá, ¿para qué me pongo a comprar eso? (Laura, segundo encuentro, julio de 2015)

Pero además de estos "efectos" cotidianos, o como parte y trasfondo de ellos, hay una demanda implícita en el trazado y la apropiación de las fronteras entre Estados, vinculada con la pertenencia, que las trayectorias migratorias tensionan con mayor o menor intensidad, y que se traduce en diversas formas de extrañamiento para el migrante ${ }^{4}$. Esa demanda, y ese extrañamiento, tienen una materialización espacio-temporal:

...una de las preguntas... que más me han hecho en este tiempo desde que me vine la primera vez es "¿y cuándo va a volver?". Entonces la gente dice "ise va a quedar para siempre en Chile?, ¿entonces ya nunca más va a volver?". O por ejemplo acá en Chile: "iotra vez te vas para Colombia?, pero si acabas de estar allá. ¿Tú no tienes trabajo acá? Pero si tú vives acá". Hay siempre un cuestionamiento en ambos países de por qué voy, por qué no me quedo (...) he tenido yo como que ir haciendo una especie como de discernimiento y también como empoderándome en mi propia movilidad o no sé cómo decirlo, o indefinición, o qué, porque tampoco le puedo llamar nomadismo, hay como dos puntos entre los cuales yo me muevo permanentemente, donde tengo afectos, pequeñas posesiones, porque no tengo en ningún lugar una casa bajo mis escrituras, pero donde tengo ropa en el armario de mi abuela, en el cajón de mi mamá... (Laura, segundo encuentro, julio de 2015) ${ }^{5}$

La angustia de Laura respecto de estas interpelaciones, el modo en que esta problematización atraviesa su trama narrativa, da cuenta cabal del trastocamiento espacio-temporal implicado en la migración, y también hace

${ }_{4}$ Es probable que los procesos migratorios que tienen una historia anterior al trazado de las fronteras geopolíticas de los Estados, es decir, parte de las migraciones fronterizas, tengan especificidades que no se ajusten a estas afirmaciones.

5 Todos los énfasis en las citas de los entrevistados son propios. 
un Ilamado a darle cabida en nuestros análisis y categorías; el modo de hacerlo debiese ser contextual. Mi intención, en todo caso, no es otra que dar cuenta de esta experimentación espacio-temporal de simultaneidad encarnada, que apareció en el trabajo de campo como indicio que exigía tener voz.

Por otra parte, y aunque quizás este tipo de señalamientos no han tenido tanta visibilidad en los replanteos que han propuesto los representantes del enfoque transnacional, algunos de ellos también han aludido a la necesidad de encontrar métodos que permitan capturar las complejas temporalidades en las que se producen algunas dinámicas migratorias, y han señalado la imposibilidad de estudiarlas solamente en un momento del tiempo, debido a que involucran procesos múltiples e interactivos más que eventos únicos (Khagram, Levitt, 2008). En todo caso, la dimensión temporal no ha estado ausente de los estudios migratorios, pero su presencia se ha vinculado más que nada a la preocupación por la posibilidad de dar cuenta del cambio social y cultural que implica la migración (Velasco, Gianturco, 2012).

Sin embargo, estos análisis temporales en general dan por sentado el carácter longitudinal del desarrollo del tiempo, y pocas veces han dado lugar a las diversas experiencias de la temporalidad que coexisten en una misma persona, que es precisamente una vivencia muy poderosa en las biografías migrantes. Aunque constituye un desafío, sería necesario reflexionar y experimentar un poco más en torno a las posibilidades de erosionar la linealidad temporal metodológica. Antonio Cornejo Polar, en su trabajo sobre el proceso de migración interna desde el área andina hacia Lima, ha puesto estas cuestiones en foco, al sostener que el migrante

no intenta sintetizar en un espacio de resolución armónica [las dos experiencias de vida, al menos, que su migración implica]; imagino -al contrario- que el allá y el aquí, que son también el ayer y el hoy, refuerzan su aptitud enunciativa y pueden tramar narrativas bifrontes y -hasta si se quiere, exagerando las cosasesquizofrénicas. (Cornejo Polar, 1996, p. 841, énfasis propio)

Para nombrar esta experiencia, el autor habla de una "heterogeneidad no dialéctica".

Esta multitemporalidad tiene diversas formas, que habría que priorizar en cada contexto de estudio. Por una parte, el migrante trae diversas experiencias de la temporalidad -por ejemplo, las de su ruralidad, o su urbanidad, las de la celebración, las de la religiosidad, las de los ritos nacionales, etc.- que se encuentran con las temporalidades del espacio al que llega. A su vez, están presentes las temporalidades de su biografía, atravesadas por la temporalidad histórica de la sociedad de la que viene, o de las sociedades en las que ha vivido. Está también la experiencia temporal de la provisoriedad con la que suele vivirse la migración, por más que esa estancia provisoria de derecho tienda, en los hechos, a transformarse en durable (Sayad, 1998). 
A esto se suma la nueva temporalidad que comienza a construirse en el proceso migratorio, que también es absolutamente personal. Para algunos la migración comienza cuando el proceso de decisión empieza a gestarse, eso puede implicar meses, semanas, días, u horas. Para otros, el hito es el registro del cruce fronterizo, o el viaje, o cierto evento en la travesía. Además, comienzan a marcarse secuencias, regularidades, intensidades, etapas, en la historia migratoria que se va construyendo en el lugar al que se llegó. En ese sentido, Benza (2000) Ilama la atención sobre el hecho que es frecuente que los migrantes se pregunten entre sí cuánto tiempo hace que llevan en el país en el que están: "Pareciera que la historia individual, desde el momento de la migración, debe ser contabilizada no sólo por la cantidad de años de edad, sino también por la cantidad de años viviendo en el nuevo espacio" (2000, p. 45). Puede que estas distinciones no sean relevantes para determinados recortes temáticos, o enfoques teóricos, u objetivos de investigación, pero es importante que podamos sopesar esta complejidad cuando puede tener implicancias en nuestros "hallazgos". Por ejemplo, en su reflexión sobre los cambios en las relaciones de género de migrantes peruanos residentes en Buenos Aires, Carolina Rosas observaba que: "Pudimos... captar el carácter procesual de las transformaciones; esto es importante, entre otras cosas, para comprender que la magnitud de los cambios en los estatus y relaciones de género depende del 'momento posmigratorio' que se analice" (2010, p. 203). Este tipo de consideraciones son decisivas en muchos casos.

Laura, con su experiencia, también cuestiona ciertas asunciones temporales convencionales en materia migratoria:

...todos esperarían que la migración es quedarte en Chile y decir "hace 10 años
no veo a mi familia", y todos "iay, qué mal!, pero acá tienes una casa y tienes un
trabajo y ya hablas como chilena", y no sé qué más cosas, y es como esa cosa de...
algunas veces me han dicho "ipero ya llevas seis años y no dices una sola palabra
chilena!", entonces también como que no sé cuáles son las expectativas que tienen
sobre uno, sobre la identidad de uno. (Laura, segundo encuentro, julio de 2015)

Hay a su vez otra arista de la temporalidad implicada de modo particular en el tema del estudio del que surge este artículo, relacionada con el carácter performático de la frontera, que se vincula en este caso con la relación ontológica entre el antes y el después, y que en los sujetos de esta investigación es doble: aparece en la experiencia de una persona con una subjetivación sexo-genérica no heteronormativa -el antes y el después de la "salida del clóset"- y en la del migrante que cruza la frontera geopolítica. En este último atravesamiento del antes-después, la persona pasa a ser un migrante, o un extranjero, se descubre en nuevas pertenencias, y se re-descubre en otras. En estas experiencias, la frontera se transforma en el instante de "envolvimiento recíproco y contradictorio del antes y el después"; como dice Eribon, "se es todavía lo que se va a dejar de 
ser y se es ya lo que se va a ser" (2001, p. 153), otro aspecto crucial de la temporalidad del proceso migratorio cuyas implicancias para ciertas temáticas es importante considerar, y que además remite de manera insistente al carácter espacio-temporal de la frontera (Mezzadra, Neilson, 2016).

La otra tensión que atravesó el proceso de investigación, y que propongo abordar aquí, se vincula con el intento de estabilizar categorizaciones respecto de las subjetivaciones sexo-genéricas, que se hizo manifiesta en una primera etapa de construcción de la muestra. En efecto, uno de los criterios de selección para esa construcción fue la decisión de excluir a la población trans e intersex, con la idea de no dificultar aún más la búsqueda de las personas con las que trabajar en la investigación, que fue uno de sus principales obstáculos. Pero, al realizar las entrevistas, supe por ejemplo que uno de los entrevistados, Marcelo (peruano, 36 años, 3 viviendo en Santiago al momento del primer encuentro, en enero de 2015), tuvo una larga experiencia trans en su vida, que en su trama narrativa termina con el cruce de la frontera, y por eso en la actualidad se define como gay; Felipe (colombiano, 19 años y cuatro viviendo en Santiago al momento de comenzar con nuestros encuentros, en julio de 2014), por su parte, se presentó como gay en todas las entrevistas, pero empezó a incursionar en experiencias trans desde sus performances de drag queen. $\mathrm{Y}$ todas las biografías traían al relato estas vivencias de tránsito, que hicieron evidente la arbitrariedad de mi categorización. "Yo te digo que soy lesbiana pero no te estoy descartando que me gusten hombres", me decía Daniela (colombiana, 29 años y dos viviendo en Santiago al momento de comenzar con las entrevistas, en abril de 2014), cuestionando el carácter esclavizante de estas categorías, aún incluso de las "no normativas" ${ }^{\prime \prime}$.

Por supuesto que el carácter procesual -es decir, contingente y contextualde las subjetividades excede su dimensión sexual y de género, pero en este caso se hizo particularmente evidente en relación con ese aspecto. Y la violencia que subyace al intento de trazar fronteras en esta dimensión vital, a la que en nuestras sociedades occidentales le hemos atribuido un carácter ontológico, se hizo patente en las etapas más iniciales de este estudio: "Usted lo que quiere es clasificarme", me acusó Martín, el primer migrante que entrevisté en la fase preliminar de este largo proceso de investigación. Ese fue el detonante más temprano de estas reflexiones en torno a las fronterizaciones epistémicas, y a la noción de frontera. "Usted quiere encasillarme", continuó Martín. Esa fue su reacción cuando, después de dos horas de una farragosa conversación, se

\footnotetext{
6 En este trabajo se entiende la normatividad del modo en que lo propone Judith Butler: "Suelo utilizar 'normativo' de una forma que es sinónima de 'concerniente a las normas que rigen el género'" (Butler, 2007, p. 25), y más específicamente, la heteronorma, porque ciertamente hay muchas otras formas de normalizar la subjetivación sexo-genérica, las que a su vez son variables en el tiempo y el espacio.
} 
atrevió a contarme que había tenido experiencias sexuales con otros hombres, y entonces le pregunté si era bisexual, pues ya me había dicho que vivía en pareja con su novia. Esta temprana interpelación fue muy movilizadora, en varios sentidos. Uno de ellos, el cuestionamiento del poder instituyente de realidades que tiene el saber científico, la arbitrariedad de esa institución, y la relación de dominación que le subyace. No son planteos novedosos, pero adquieren otra dimensión cuando a través de la interpelación es uno mismo el que es colocado en el lugar del que ejerce esa violencia. Por otra parte, me confrontó con la contradicción que subyacía a mi comentario, pues partiendo desde la perspectiva de la performatividad del género, con el uso del verbo "ser" al "categorizarlo" como bisexual estaba pretendiendo estabilizar un proceso de subjetivación por definición inacabado, es decir, estaba tratando de trazar una frontera nítida allí donde aquello que se intentaba contener, se desbordaba por una porosidad inasible.

\section{Una mirada intersticial en torno a las fronteras}

Las reflexiones previas ponen en un primer plano la necesidad de replantearse la idea de frontera pensada en términos amplios, es decir, como artefacto conceptual-metodológico, dado que en la actualidad prevalece un nivel de confusión importante sobre esta noción. Como explica Grimson, es preciso ser cuidadoso con este concepto: "No sólo porque no todo es una 'frontera', sino porque no debemos pedirle que haga más trabajo teórico del que puede realizar" (Grimson, 2003, p. 21). Otro riesgo reside en el hecho que, como señala Vila (2001), con esta proliferación de la noción de frontera, que implica utilizarla para referirse a cualquier tipo de situación en la que la idea de límite esté presente -entre países, géneros, grupos étnicos, disciplinas, etc.-, se tiende por una parte a igualar experiencias que pueden ser muy diferentes -como cruzar fronteras entre países o grupos étnicos-, y por el otro, a homogeneizar las propias fronteras. Estas advertencias, entonces, deben implicar una permanente vigilancia epistemológica.

Ahora bien, esta confusión que prima en torno al concepto de frontera es proporcional a su potencial heurístico. En ese marco, la propuesta es abandonar la dicotomía restricción/flujo que ha primado en las aproximaciones y debates en torno al concepto de frontera en el campo migratorio. Es decir, no pensarla, o no pensarla solamente, como un límite sedimentado (histórico) -el lado de la estructura- (ya sea que ese límite esté trazado entre países o ciudades, entre sexos, entre géneros, entre "razas", etnias, clases, grupos etarios), ni como un espacio de flujos, de pasos y pasajes que la trastocan -el lado de la agencia-, sino como un intersticio, es decir, como una hendidura, un poro. Eso significa dejar de pensar la frontera como un "en" o como un "a través", y pensarla en cambio como un "entre". 
Esta imagen del intersticio es particularmente útil, porque la hendidura, para producirse, requiere necesariamente esa condensación, esa sedimentación que supone el límite, que es la que se horada, pero a la vez implica la posibilidad de romper, trastocar, debilitar esa condensación. Entonces, esa posición permite pensar la frontera como estructura y agencia a la vez (Guizardi et al., 2015), y así captar de modo más cabal las experiencias de los entrevistados de esta investigación, por ejemplo, cuando a la vez que re-producen en sus cuerpos y vivencias los controles sobre la sexualidad que se gestaron en el allá (particularmente en la familia de origen), utilizan estratégicamente herramientas que le proporciona el acá para desafiar ese control, ya sea sutilmente o de modo más radical. Y pensar a la frontera como estructura y agencia a la vez a partir de una experiencia en particular también hace posible un cambio epistemológico necesario: "abandonar la pretensión de encontrar una respuesta trascendente a la pregunta por la relación entre el sujeto y la estructura, y asumir que la respuesta es casuística" (Grimson, 2012, p. 35).

Pensar/imaginar la frontera de modo intersticial -quitándole, de paso, calidad ontológica a aquello que la frontera separa- pone el acento en las posibilidades erosivas y corrosivas (Grimson, 2012) que el cruce de la frontera (sea del tipo que fuere) habilita; permite pensar su atravesamiento como potencialmente desarraigante -usando esta palabra sin connotación territorial-. La idea de "acciones corrosivas" se refiere específicamente "a los agenciamientos sociales y culturales que apuntan a provocar la ruptura, la elaboración o la disolución de sedimentos concretos" (Grimson, 2012, p. 167), sean los sedimentos políticos del Estado, los de una configuración cultural nacional, los que genera la norma hegemónica para la subjetivación sexo-genérica, los sedimentos de las fronteras que separan el campo de la ciudad, o el interior de la capital, e incluso los sedimentos de la propia distinción origen/destino, los que se condensan en fronteras dentro de la propia ciudad que habita-vive el migrante, entre otros.

La propuesta es pensar a la frontera, entonces, despojada de su sentido topográfico, como una "zona intersticial" (Gupta, Ferguson, 2008), como espacio para la fluidez, para la libertad de movimiento gracias al despojo de la sobrecarga cultural que permite ese gesto de horadar, como posibilidad de exploración creativa por los dislocamientos situacionales y las combinaciones innovadoras a las que abre paso (Hannerz, 1997). Esta mirada permite incluso recuperar la dimensión lúdica y gozosa de la frontera.

Pero a la vez esta imagen intersticial, esta idea de hendidura mediando entre espacios-tiempos (materiales y simbólicos), implica no perder de vista esa densa solidificación histórica que se trata de horadar. Y eso es relevante para no caer en la visión "romántica" de la creatividad y fluidez de la frontera como metáfora de las "identidades lábiles", que podría llevar a soslayar la crudeza 
y conflictividad que implica su atravesamiento -tanto de las fronteras físicas como de las simbólicas- (Caggiano, 2003). Es decir, no se trata simplemente de celebrar la porosidad, maleabilidad y contingencia de las fronteras, porque no es posible desoír la conflictividad que hay implicada en su atravesamiento. El poder de las sedimentaciones que la han instituido puede dejarse caer con su peso punitivo de diversas formas.

Aludiendo específicamente a las fronteras geopolíticas, Grimson sostiene que la metáfora del "cruzador de fronteras" como nuevo sujeto de la historia tiende a invisibilizar el conflicto que suponen las asimetrías entre sectores, grupos y Estados (Grimson, 2012). Es preciso, entonces, no perder de vista que las fronteras son producto de acciones humanas sedimentadas, y esa sedimentación, aunque horadable, puede ser muy densa y potente. No por arbitrarias (por históricamente construidas) las fronteras dejan de ser poderosamente eficaces (Caggiano, 2003). Por lo tanto, cruzar una frontera no significa desdibujarla (Grimson, 2003), muy por el contrario, muchas veces implica transformarse uno mismo en frontera (Balibar, 2005). Pero como el mismo Grimson señala, esa sedimentación poderosa de las fronteras también pone en evidencia su humanidad, su construcción cotidiana (Grimson, 2000) y, por lo tanto, su contingencia, historicidad y fragilidad.

Esta tensión entre estructura y agencia en la propia definición de frontera tiene su correlato en el modo de pensar al sujeto que la atraviesa. Es decir, así como la frontera puede entenderse como estructura y agencia a la vez, el sujeto que la atraviesa debe pensarse igualmente como reforzador y como cruzador de fronteras a la vez (Vila, 2007), pues las fronteras son "instituciones sociales complejas, que están marcadas por tensiones entre prácticas de reforzamiento y prácticas de atravesamiento" (Mezzadra, Neilson, 2016, p. 22). Sumado a este aspecto, Vila hace un alcance de mucha relevancia, al sostener que las "fronteras divergentes adquieren un peso específico distinto en relación a las diversas posiciones de sujeto (y las diferentes narrativas que tratan de dar cuenta de tales posiciones) que dichos actores deciden usar en sus procesos identificatorios" (Vila, 2007, p. 55). En el estudio del que surgió esta reflexión se pudo observar que, mientras que en determinadas situaciones son las fronteras sexo-genéricas las que adquieren relevancia en las narrativas de los entrevistados, en otras priman las fronteras nacionales, o las de clase. Además, estas observaciones respecto del sujeto que cruza la frontera revelan otra arista decisiva del concepto: el hecho de que la frontera produce subjetividad (Mezzadra, Neilson, 2016).

Hay una última precisión sobre este constructo general de frontera que es importante realizar: es necesario quitar de la idea de intersticio su anclaje exclusivamente territorial, como ya he sugerido implícitamente. En cambio, es preciso que pensemos en un intersticio espacio-temporal. Recuperar la 
dimensión temporal es una tarea imprescindible -aunque dificultosa-, porque es una arista que se ha soslayado en las discusiones sobre frontera, y la experiencia migratoria supone un dislocamiento temporal (además de espacial) de la biografía que tiene implicancias decisivas en los modos complejos de experimentar las fronteras de los migrantes.

En el caso de estudio que se comenta en este artículo, esta mirada intersticial de la frontera permitió, por ejemplo, arribar a la noción de extrañamiento como una idea clave para explicar los hallazgos. Ese extrañamiento, es decir, ese estar en el entre, sin pertenecer completamente ni al país de origen ni al de destino, hace posible al migrante sacudirse ciertos lastres culturales normativos en el proceso de subjetivación sexo-genérico. Así, el cuerpo puede liberarse de algunas ataduras generadoras de sufrimiento social, alivianarse para una experiencia vital más gozosa, que puede ser más intensa que las nuevas constricciones que le imponen las cartografías de la extranjeridad, la nacionalidad y la clase propias de ese nuevo "mapa social".

Esa experimentación del "estar entre", esa posición intersticial, esa no pertenencia plena ni a la configuración cultural de la que se partió ni a aquella a la que se llegó, es la que precipita ciertas acciones corrosivas, ciertos agenciamientos temporales, que permiten renegociar las fronteras a partir de las que se van construyendo las subjetividades. Y estas prácticas de subjetivación pueden entenderse entonces como gestos de autonomía (Rosas, 2010), esfuerzos emancipatorios que marcan estos procesos migratorios, tácticas que hacen parte de una micropolítica de la vida cotidiana en la que estas migraciones jugaron un rol significativo.

\section{Consideraciones finales}

El derrotero de este artículo propuso una reflexión en torno a ciertas tensiones experimentadas en el trabajo de campo de una investigación sobre procesos de subjetivación sexo-genéricos de migrantes de origen colombiano y peruano residiendo en Santiago de Chile, tensiones con derivas metodológicas, teóricas y epistemológicas. La tensión aludida se vincula con la arbitrariedad experimentada al intentar trazar fronteras claras y estables (operacionalizables) para definir los sujetos de la investigación, en relación con dos de sus atributos: la relación tiempo-espacio del desplazamiento migratorio, y la forma de subjetivación sexo-genérica.

Problematizar esas fronteras específicas, debido a la experimentación espacio-temporal de simultaneidad encarnada que se evidenciaba en estas biografías migrantes, y al carácter procesual (contingente y contextual) de sus subjetivaciones sexo-genéricas, nos condujo a proponer, a partir de la revisión de aproximaciones de otros autores, una noción de frontera como intersticio 
espacio-temporal, como un modo de intentar hacerse cargo de la violencia epistémica supuesta en el trazado de esas fronteras.

Pensar la frontera como intersticio, según se propuso, nos confrontó con replanteamientos metodológicos para investigar las migraciones -icómo operacionalizar la ubicuidad espacio-temporal de los desplazamientos?-, cuestionamientos epistemológicos -ipor qué tendemos a atribuir un carácter ontológico a las categorizaciones que definimos al trazar fronteras?--, y teóricos, en la medida que esta mirada intersticial nos interpeló a pensar los procesos sociales en ese poroso lugar-tiempo que media entre estructura y agencia. Podría decirse, entonces, que este ejercicio reflexivo nos sugirió un extrañamiento del propio trabajo investigativo, o sea, una invitación a posicionarnos intersticialmente entre las categorías que construimos en el trabajo analítico y la asunción de la necesaria y permanente inestabilidad de esas categorías, de modo de poder advertir lo que siempre, inevitablemente, se escapa de esas solidificaciones, y rebaza por las porosidades de toda frontera, o de todo tipo de fronteras.

\section{Referencias bibliográficas}

BALIBAR, Étienne. ¿Qué es la frontera? In: BALIBAR, Étienne. Violencias, identidades y civilidades. Para una cultura política global. Barcelona: Gedisa, 2005, p. 77-86. BENZA, Silvia. Migración de ciudadanos peruanos hacia Argentina: cruces fronterizos, períodos intermedios, liminalidad y communitas. Revista de Investigaciones Folclóricas, n. 15, p. 42-48, 2000.

BOURDIEU, Pierre. ¿Qué es lo que hace a una clase social? Acerca de la existencia teórica y práctica de los grupos. Revista Paraguaya de Sociología, n. 31, n. 89, p. 7-21, 1994.

BUTLER, Judith. El género en disputa. El feminismo y la subversión de la identidad. Barcelona: Paidós, 2007.

CAGGIANO, Sergio. Fronteras múltiples: Reconfiguración de ejes identitarios en migraciones contemporáneas a la Argentina. Cuadernos del IDES, n. 1, 2003.

CORNEJO POLAR, Antonio. Una heterogeneidad no dialéctica: sujeto y discurso migrantes en el Perú moderno. Revista Iberoamericana, n. 176-177, p. 837-844, 1996.

DOMENACH, Hervé; PICOUET, Michel. El carácter de reversibilidad en el estudio de la migración. Notas de Población, n. 49, p. 49-69, 1990.

ERIBON, Didier. Reflexiones sobre la cuestión gay. Barcelona: Anagrama, 2001.

GRIMSON, Alejandro. El puente que separó dos orillas. Notas para una crítica del esencialismo de la hermandad. In: GRIMSON, Alejandro. Fronteras, naciones e identidades. La periferia como centro. Buenos Aires: Ciccus-La Crujía, 2000, p. 201-231. 
GRIMSON, Alejandro. Disputas sobre las fronteras. Introducción a la edición en español. In: SCOTT, Michaelsen; JOHNSON, David E. Teoría de la frontera. Los límites de la política cultural. Barcelona: Gedisa, 2003, p. 13-23.

GRIMSON, Alejandro. Los límites de la cultura. Crítica de las teorías de la identidad. Buenos Aires: Siglo XXI, 2012.

GUIZARDI, Menara Lube; VALDEBENITO, Felipe; LÓPEZ, Eleonora; NAZAL, Esteban. Condensaciones en el espacio hiperfronterizo: Apropiaciones migrantes en la frontera norte de Chile. In: GUIZARDI, Menara Lube (ed.). Las Fronteras del Transnacionalismo. Límites y desbordes de la experiencia migrante en el centro y norte de Chile. Santiago: Universidad de Tarapacá y Ocho Libros, 2015, p. 224-257.

GUPTA, Akhil; FERGUSON, James. Más allá de la "cultura": espacio, identidad y las políticas de la diferencia. Antípoda, n. 7, p. 233-256, 2008.

GUTIÉRREZ VIDRIO, Silvia. El análisis del discurso: aportes teórico-metodológicos para el estudio de la migración. In: ARIZA, Marina; VELASCO, Laura. Métodos cualitativos y su aplicación empírica: por los caminos de la investigación sobre migración internacional. México, DF: UNAM, Instituto de Investigaciones Sociales y El Colegio de la Frontera Norte, 2015, p. 353-384.

HANNERZ, Ulf. Fluxos, fronteiras, híbridos: palavras-chave da antropologia transnacional. Maná, v. 3, n. 1, p. 7-39, 1997.

HERRERA, Gioconda; NYBERG SØRENSEN, Ninna. Migraciones internacionales en América Latina: miradas críticas a la producción de un campo de conocimientos. Íconos. Revista de Ciencias Sociales, n. 58, p. 11-36, 2017.

HILLER, Renata. Desnaturalizar los vínculos entre conyugalidad y ciudadanía. El matrimonio en Argentina, su trayectoria y los cambios recientes. In: MORÁN FAÚNDES, José Manuel; SGRÓ RUATA, María Candelaria; VAGGIONE, Juan Marco. Sexualidades, desigualdades y derechos. Reflexiones en torno a los derechos sexuales y reproductivos. Córdoba: Ciencia, Derecho y Sociedad, 2012, p. 227-250.

KHAGRAM, Sanjeev; LEVITT, Peggy. Constructing Transnational Studies. In: KHAGRAM, Sanjeev; LEVITT, Peggy. The Transnational Studies Reader. New York: Routledge, 2008, p. 1-18.

LEVITT, Peggy; JAWORSKY, B. Nadya. Transnational Migration Studies: Past Developments and Future Trends. Annual Revue of Sociology, n. 33, p. 129156, 2007.

MAGLIANO, María José. Interseccionalidad y migraciones: potencialidades y desafíos. Estudos Feministas, v. 23, n. 3, p. 691-712, 2015.

MEZZADRA, Sandro; NEILSON, Brett. La frontera como método. O la multiplicación del trabajo. Buenos Aires: Tinta Limón, 2016.

PECHENY, Mario. Introducción. Investigar sobre sujetos sexuales. In: PECHENY, Mario; FIGARI, Carlos; JONES, Daniel. Todo sexo es político: estudios sobre sexualidad en Argentina. Buenos Aires: Libros del Zorzal, 2008, p. 9-17. 
ROSAS, Carolina. Implicaciones mutuas entre el género y la migración mujeres y varones peruanos arribados a Buenos Aires entre 1990 y 2003. Buenos Aires: EUDEBA, 2010.

SAYAD, Abdelmalek. A Imigração ou os Paradoxos da Alteridades. São Paulo: Editora da Universidade de São Paulo, 1998.

STANG, Fernanda. Fronteras, sexualidades y procesos de subjetivación. Migrantes LGTBIQ colombianos y peruanos en Santiago de Chile. (Tesis para optar al grado de Doctora en Estudios Sociales de América Latina). Córdoba: Universidad Nacional de Córdoba, 2018.

VELASCO, Laura; GIANTURCO, Giovanna. Migración internacional y biografías multiespaciales: una reflexión metodológica. In: ARIZA, Marina; VELASCO, Laura. Métodos cualitativos y su aplicación empírica. Por los caminos de la investigación sobre migración internacional. México, DF: Instituto de Investigaciones Sociales, UNAM y El Colegio de la Frontera Norte, 2012, p. 115-150.

VILA, Pablo. Versión estadunidense de la teoría de frontera: una crítica desde la etnografía. Papeles de Población, v. 7, n. 30, p. 11-30, 2001. 